\title{
The Analysis of the Treatment Outcomes of Proximal Humeral Fractures with Locking Plates
}

\author{
Kwang-Won Lee, Yoon-Sub Hwang, Choon-Myeon Kim, Dae-Suk Yang, Tae-Soo Park ${ }^{1}$ \\ Department of Orthopedic Surgery, Eulji University College of Medicine, Daejeon, ${ }^{1}$ Department of Orthopaedic Surgery, Hanyang University Guri Hospital, \\ Hanyang University College of Medicine, Guri, Korea
}

Background: The aim of this study was to assess the clinical outcomes after treatment of proximal humeral fractures with locking plates, and to determine which factors influence the clinical and radiological outcomes.

Methods: Fifty six patients who were treated with locking plates for proximal humeral fractures and had been followed for more than 1 year were enrolled in this study. We performed functional evaluation using the Constant score and analyzed radiographic results. The following factors that may potentially influence the clinical outcomes were assessed: age, gender, type of fracture, presence of medial metaphyseal comminution, bone mineral density, anatomical reduction, restoration of medial mechanical support, and postoperative complications.

Results: The mean Constant score was 70.1 points at the final follow-up. Female gender, 4-part fractures, AO type-C fractures, and fractures with medial metaphyseal comminution were associated with a poor clinical outcome. On the other hand, restoration of medial mechanical support and accurate anatomical reduction had a positive influence on clinical outcomes. Postoperative complications resulted in 3 patients (intra-articular screw perforation: 1 patient, varus deformity with screw loosening: 1 patient, nonunion: 1 patient). Conclusions: When treating proximal humeral fractures with locking plate fixation, following factors: a female gender, Neer type 4-part fracture, AO type $\mathrm{C}$ fracture, and medial metaphyseal comminution are important risk factors that surgeons should take into consideration. Factors that contribute to better clinical outcomes of operative treatment for humeral fractures are accurate anatomical reduction and restoration of medial mechanical support.

(Clin Shoulder Elb 2014;17(1):10-17)

Key Words: Humerus; Proximal humeral fracture; locking plates

\section{Introduction}

Fractures of the proximal humerus comprise around 4-5\% of all fractures. In general, for the treatment of nondisplaced fractures, conservative treatment leads to a satisfactory outcome, but for displaced and unstable fractures, surgical intervention is usually required to gain a functional recovery. ${ }^{1,2)}$ The increase in the aging population and the incidence of osteoporosis in these populations has in turn increased the prevalence of the proximal humeral fractures. Especially, the need for operative treatment is increasing with rising numbers of the complex fractures. ${ }^{3)}$

The various methods to treat fractures of the proximal hu- merus are percutaneous cerclage wiring, tension band wiring, intramedullary nailing, plating, and humeral head replacement. Of these, recent clinical data have shown favorable results for the treatment of fractures with locking plates. As compared to the conventional plate fixation methods, this technology is able to maintain blood supply to the region of fracture, achieve higher success rate of fixation at the interface between the plate and the locking screws, can fix the plates at multiple angles, and gain both angular and rotational stability at the point of fixation. Thus, the method using locking plates has been extensively used in many orthopedic clinics. ${ }^{4,5)}$ Successful clinical outcomes using the locking plates in treating proximal humeral fractures have

Received August 16, 2013. Revised December 14, 2013. Accepted January 20, 2014.

Correspondence to: Tae-Soo Park

Department of Orthopedic Surgery, Hanyang University Guri Hospital, 153 Kyungchoon-ro, Guri 471-701, Korea

Tel: +82-31-560-2310, Fax: +82-42-259-1289, E-mail: poxkoreana@gmail.com

Financial support: None. Conflict of interests: None. 
been shown by several studies. ${ }^{4,-68)}$ The clinical outcomes were satisfactory even in comminuted fractures or severe osteoporosis. ${ }^{9,10)}$ Nevertheless, there were some cases of inability to maintain anatomical reduction leading to post-surgical complications such as malunion of fracture. ${ }^{11}$

This study analyzed the clinical and radiological outcomes of locking plate fixation for proximal humeral fractures. We aimed to determine factors that influence the clinical outcomes of this method, and find out which factors are important for successful clinical outcomes.

\section{Methods}

\section{Subjects of Study}

From Dec 2005 to Dec 2010, 56 patients with fractures of the proximal humerus underwent operative fixation using locking plates. Patients who were able to participate in the final follow-up visit for more than a year were selected for the study. The average age of the patient was 57.6 years (range; 24 to 84 years), and of these, 22 patients were more than 65 years of age and 34 patients were below. The study comprised of 14 male and 42 female patients. The mean follow-up period was 15.3 months (range, 12 to 36 months). Grading of the fracture pattern was accomplished radiographically using the Neer classification system. According to this classification, 27 patients had 2-part fractures, 21 patients had 3-part fractures, and 8 patients had 4-part fractures. Grading of the fracture pattern was also determined through the $\mathrm{AO}$ classification, by which 14 patients had an A-type fracture, 28 patients a B-type, and 14 patients a C-type. Patients that had a previous history of fractures of the shoulder either on the affected or the unaffected side were excluded from the study. Other exclusion criterion from the study was if hemiarthroplasty was performed. Hemiarthroplasty was performed in patients with severe osteoporotic 3- or 4-part fracture and/or dislocation, and head splitting fractures.

\section{Surgical Methods and Postoperative Rehabilitation}

Operative fixation using locking plate was used to treat patients with 2-part, 3-part, and 4-part fractures. Patients with 2-part fractures were only included for surgery with locking plates if they showed severe displacement that made conservative treatment difficult. All surgical treatment was performed in 4.5 days on the average (range; 1 to 15 days) after the injury by one skilled orthopedic surgeon. The locking plates used were either LPHP ${ }^{\circledR}$ (Locking Proximal Humerus Plate; Synthes, Oberdorf, Switzerland) or PHILOS ${ }^{\circledR}$ (Proximal Humerus Internal locking System; Synthes, Mezzovico, Switzerland). LPHP ${ }^{\circledR}$ was used for the first 22 patients that underwent surgery at our clinic, and PHILOS $^{\circledR}$ was used for the later 34 patients.

Patient was placed into a beach chair position under general anesthesia. A deltopectoral approach was chosen for exposure of all fractures. After open reduction of the fracture, the locking plate was fixed to the humerus using locking screws. If a fracture with medial metaphyseal comminution was present, the locking screws were fixed to the proximal humeral calcar, and if needed additional allogenous bone grafts were used. In a patient with severe defect of the posteromedial cortical bone, femoral head strut allograft was used to stabilize the anatomical reduction. Post-operatively, a shoulder abduction brace was applied for 4 weeks. Pendulum exercise and passive forward flexion was be-
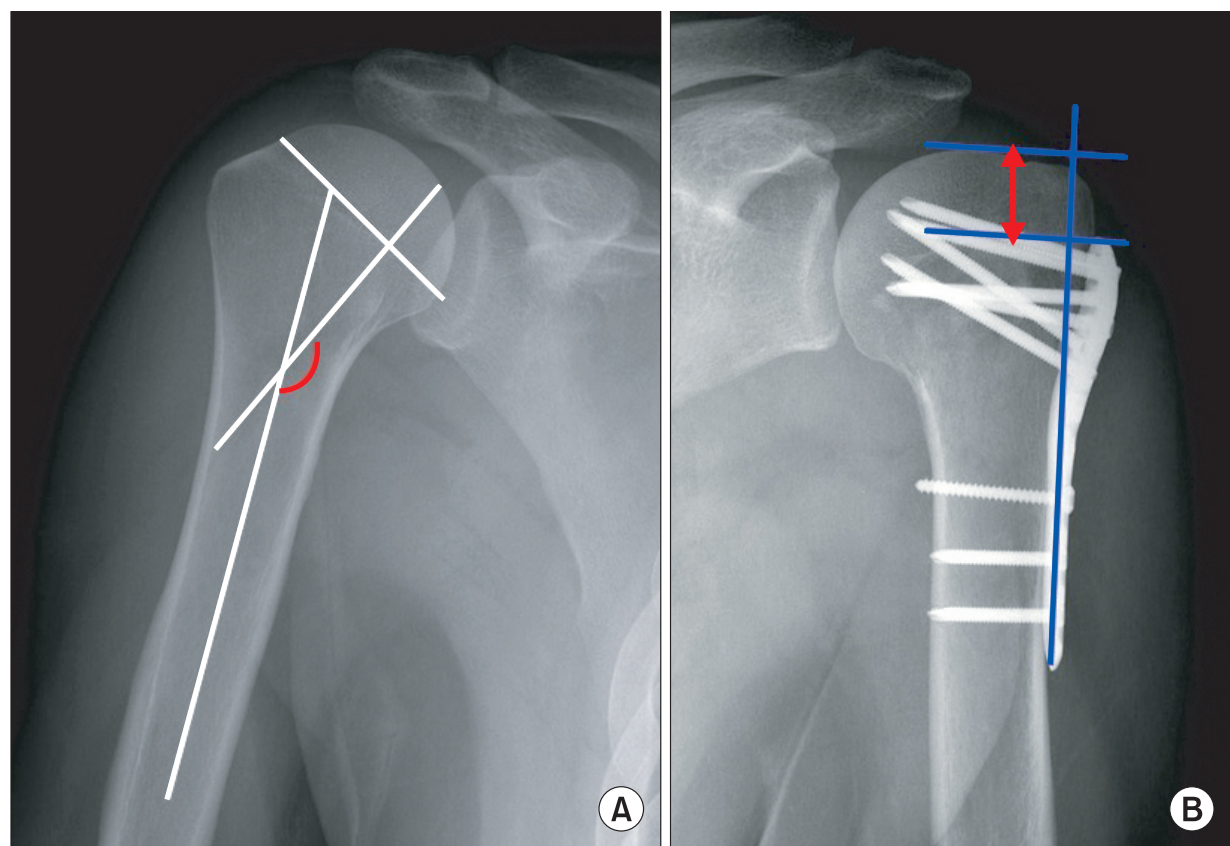

Fig. 1. Radiographic measurements in the true AP view of the shoulder. (A) The angle between the line bisecting the humeral shaft and the line perpendicular to the line from the superior border to the inferior border of the articular surface was measured as the humeral neck shaft angle (semicircle). (B) Humeral head height (line with arrows) was measured as the distance from the top of the plate to the top of the humeral head, both were measured perpendicular to the axis of the plate. 
gun 1 week after operation. Active range of motion exercise was begun when bony union was identified on radiographs.

\section{Assessment of Clinical Outcomes}

The clinical outcomes of the operation were assessed using Constant score, which comprises of the following assessment variables: pain score (15 points), functional assessment (20 points), range of motions (40 points), and muscle strength (25 points). The Constant score was graded as 'excellent' (86-100 points), 'good' ( $71-85$ points), 'moderate' (56-70 points), or 'poor' (0-5 5points). ${ }^{12)}$

The pre-operative radiographic assessment was carried out using plain radiographs. The post-operative plain radiographs were taken immediately postoperative, 2-weeks, 6-weeks, 3-months, 6-months and 1-year after operation. The anteroposterior, true anteroposterior, lateral, and axillary view of the shoulder joint were taken. At each radiographic assessment, the presence or absence of bony callus, disappearance of fracture lines, and the union of the displaced bone were examined to measure the progression of bony union. The neck-shaft angle, another indicator of treatment outcome, was measured by measuring the angle between the line perpendicular to the anatomical neck and the line parallel to the humeral shaft (Fig. 1A). The neck-shaft angle was graded radiographically according to Paavolainen et al.'s method as "good" $\left(121^{\circ}-140^{\circ}\right)$, "fair" $\left(100^{\circ}-120^{\circ}\right)$, or "poor" (less than $\left.100^{\circ}\right){ }^{13)}$ Further, the accuracy of anatomical reduction was assessed by confirming whether the medial side of the fracture achieved anatomical reduction. The degree of post-operative loss of reduction was measured by comparing the humeral neck-shaft angles and the humeral head height at immediate postoperative and at final follow-up on anteroposterior radiographs (Fig. 1B). The humeral head height, measured following Gardner et al.'s method, ${ }^{14)}$ is the distance from the top of the plate to the top of the humeral head, which are both measured perpendicular to the axis of the plate. All radiographic assessments were made twice on separate occasions by two orthopedic surgeons, who did not have access to individual radiograph details of patients. The intra-observer and the inter-observer reliability were determined using the Intraclass Correlation Coefficients (ICCs) that were established by Shrout and Fleiss. ${ }^{15)} \mathrm{ICC}$ has a value ranging from 0 , which means there is no agreement between the observers, to 1 , which means we see perfect agreement between the observers. An ICC value of $0.00-0.39$ was considered as 'poor', $0.40-0.74$ as 'moderate', and $0.75-1.00$ as 'excellent'.

We assessed the factors related to the patient and fracture status that may potentially influence the treatment outcomes of proximal humeral fractures using locking plates. These factors were patient's age, sex, presence of comorbidities such as osteoporosis, fracture type, and presence or absence of medial metaphyseal comminution. Other factors, which related to the surgical method we assessed were accuracy of the anatomical reduction and restoration of medial mechanical support of the proximal humeral fracture. Presence of osteoporosis was determined pre-operatively by calculating the bone density of the lumbar spine and hip joints using Dual Energy X-ray Absorptiometry (DEXA). Using DEXA, osteoporosis was diagnosed if a patient had a T-score of less than -2.5. The fracture type was categorized using Neer classification and AO classification systems. The anatomical reduction was deemed accurate if the difference in neck-shaft angle between that of the affected side and the opposite side was below $5^{\circ}$ after surgery, and inaccurate if the angle was above $5^{\circ}$ after surgery (standard deviation $=1$ ). The medial mechanical support of the proximal humeral fracture was deemed restored if there was cortical contact of the proximal humerus or if the proximal fragment or the femoral bone allograft was impacted, in the case where the inferomedial part of the proximal humeral fracture was fixed using locking screws. ${ }^{14,16)}$ Postoperative complications such as screw loosening, nonunion, and avascular necrosis of the humeral head were assessed by plain radiographs.

All statistical analysis was performed using a statistics program to evaluate the relationship between the factors concerning clinical and radiographic findings, such as age, sex, bone density, fracture type, presence of medial comminution of the humerus, and medial mechanical support of the fracture. Test of normality was performed to confirm a normal distribution of results. Then, results were analyzed with an independent T-test and one-way analysis of variance. Scheffe's test was used as the post-hoc comparison test. Statistical significance was set at $p<0.05$.

\section{Results}

We found that bony union of fracture takes on an average 13.6 weeks. At final follow-up, the average Constant score was 70.1 (range; 40-100). Nine patients had a score of 'excellent' (16\%), 28 patients 'good' (50\%), 13 patients 'moderate' $(23 \%)$, and 6 patients 'poor' (11\%), giving an overall $66 \%$ of patients who had a score of greater than 'good'. At final followup, the average humeral neck-shaft angle was $130.4^{\circ}$ (range, $111^{\circ}-139^{\circ}$ ). When the neck-shaft angles were classified based on the method by Paavolainen et al., ${ }^{13)} 54$ patients had a score of 'excellent', 2 patients 'good', and none 'poor'.

The postoperative humeral neck-shaft angle was on average $6.2^{\circ}\left(\right.$ range, $\left.1^{\circ}-19^{\circ}\right)$ different relative to the unaffected contralateral shoulder. Further, the humeral neck-shaft angle at final follow-up was on average $5.7^{\circ}\left(\right.$ range, $1^{\circ}-21^{\circ}$ ) lower than that at immediate post-operation. The change in humeral head height at immediate postoperative and at final follow-up was on average $1.6 \mathrm{~mm}$ (range, $0.1-7.2 \mathrm{~mm}$ ).

The change in Constant score, the humeral neck-shaft angle, and the humeral head height at immediate postoperative and at 
Table 1. Comparison of Constant Scores and Radiographic Measurements according to Factors Relating to the Patient

\begin{tabular}{|c|c|c|c|c|}
\hline & & $\begin{array}{c}\text { Constant score at last follow-up, } \\
\text { points (range) }\end{array}$ & $\begin{array}{c}\text { Changes in neck-shaft angle } \\
\text { degrees (range) }\end{array}$ & $\begin{array}{c}\text { Changes in humeral head height }{ }^{*} \text {, } \\
\text { mm (range) }\end{array}$ \\
\hline \multirow[t]{3}{*}{ Age, years } & $<65(\mathrm{n}=34)$ & $72.4(48-100)$ & $4.5(1-15)$ & $1.3(0-4.8)$ \\
\hline & $\geq 65(\mathrm{n}=22)$ & $66.4(40-87)$ & $7.5(1-21)$ & $2.1(0.1-7.2)$ \\
\hline & $p$-value & $0.100^{\dagger}$ & $0.010^{\dagger}$ & $0.092^{\dagger}$ \\
\hline \multirow[t]{3}{*}{ Gender } & Male $(\mathrm{n}=14)$ & $77.4(48-100)$ & $3.4(1-9)$ & $1.0(0.1-3.8)$ \\
\hline & Female $(n=42)$ & $67.6(40-90)$ & $6.4(1-21)$ & $1.8(0-7.2)$ \\
\hline & $p$-value & $0.017^{\dagger}$ & $0.003^{\dagger}$ & $0.045^{\dagger}$ \\
\hline \multirow[t]{4}{*}{ Neer classification } & 2 part $(\mathrm{n}=27)$ & $75.3(41-100)$ & $4.7(1-11)$ & $1.1(0-3.8)$ \\
\hline & 3 part $(n=21)$ & $67.5(40-90)$ & $5.9(1-21)$ & $1.8(0.1-7.2)$ \\
\hline & 4 part $(n=8)$ & $59.3(48-74)$ & $8.4(3-15)$ & $2.4(0.4-3.7)$ \\
\hline & $p$-value & $0.011^{ \pm \S}$ & $0.093^{\ddagger}$ & $0.092^{\ddagger}$ \\
\hline \multirow[t]{4}{*}{ AO classification } & $\mathrm{A}(\mathrm{n}=14)$ & $76.9(41-100)$ & $4.1(1-11)$ & $1.1(0-3.5)$ \\
\hline & $B(n=28)$ & $70.0(52-90)$ & $5.9(1-21)$ & $1.5(0.1-4.9)$ \\
\hline & $C(\mathrm{n}=14)$ & $63.4(40-88)$ & $6.9(1-14)$ & $2.3(0.1-16)$ \\
\hline & $p$-value & $0.026^{\ddagger \S}$ & $0.197^{\ddagger}$ & $0.126^{\ddagger}$ \\
\hline \multirow[t]{3}{*}{ Medial metaphyseal comminution } & Yes $(\mathrm{n}=28)$ & $64.6(40-87)$ & $6.8(1-21)$ & $2.1(0.1-7.2)$ \\
\hline & No $(n=28)$ & $75.5(41-100)$ & $4.6(1-11)$ & $1.0(0-3.7)$ \\
\hline & $p$-value & $0.002^{\dagger}$ & $0.050^{\dagger}$ & $0.006^{\dagger}$ \\
\hline \multirow[t]{3}{*}{ Bone mineral density, T-score } & $>-2.5(\mathrm{n}=15)$ & $70.4(41-90)$ & $5.7(1-21)$ & $1.8(0.2-4.9)$ \\
\hline & $\leq-2.5(\mathrm{n}=23)$ & $67.7(40-88)$ & $5.7(1-14))$ & $1.7(0.1-7.2)$ \\
\hline & $p$-value & $0.545^{\dagger}$ & $0.997^{\dagger}$ & $0.802^{\dagger}$ \\
\hline
\end{tabular}

${ }^{*}$ The changes in humeral neck shaft angles or humeral head heights between immediate postoperative and last follow-up radiographs. ${ }^{\dagger}$ Independent $\mathrm{t}$-test. ${ }^{\ddagger}$ ANOVA test. ${ }^{8}$ Significance determined by analysis of variance with Scheffe's post hoc method.

final follow-up in relation to the patient's age, sex, presence of medial metaphyseal comminution, and bone density has been summarized in Table 1. The humeral neck-shaft angle was decreased in patients above the age of 65 more significantly than those under the age of $65(p=0.010)$. Conversely, the Constant score was tended to be lower in patients under the age of 65 than those above, but this difference was not statistically significant $(p=0.100)$. Further, women showed a significantly lower Constant score, a higher change in humeral neck-shaft angle and humeral head height compared to men $(p<0.050)$. In terms of the fracture classification, those with a Neer 4-part fracture had a significantly lower Constant score compared to those with a Neer 2-part fracture $(p=0.011)$. Although a greater decrease in the humeral neck-shaft angle and humeral head height was seen in patients with a 4-part fracture than in patients with a 2-part fracture, there was no significant difference $(p>0.050)$. If the Constant scores of patients with 4-part fractures were excluded, the average Constant score increased to 73.1 points, which increased the percentage of total patients with a score of or above 'good' to $77 \%$. Likewise, the AO classification showed that pa- tients with C-type fractures had a significantly lower Constant score compared to patients with A-type fractures $(p=0.026)$. Again, although the humeral neck-shaft angle and humeral head height decreased more in patients with a C-type fracture than in patients with a A-type fracture, there was no significant difference $(p>0.050)$. Furthermore, if a comminuted fracture on the medial humerus was present, the Constant score was lower than if it was not $(p=0.02)$, and the change in humeral neck-shaft angle $(p=0.050)$ and humeral head height $(p=0.006)$ was greater. The Constant score, neck-shaft angle, and humeral head height in terms of bone density did not show any significant difference.

The inter-observer and intra-observer reliability were assessed by comparing the results of ICCs of measurements taken on two separate occasions by two observers. The inter-observer variation was $0.80-0.92$, whereas the intra-observer variation was $0.88-0.96$, thus giving almost the same results.

Radiographs taken immediately postoperative was used to measure the difference between the humeral neck-shaft angle of the affected and the unaffected shoulder. Compared to when the difference was greater than $5^{\circ}$, there were better clinical 
Table 2. Comparison of Constant Scores and Radiographic Measurements according to Factors Relating to the Surgery

\begin{tabular}{|c|c|c|c|c|}
\hline & & $\begin{array}{l}\text { Constant score, points } \\
\text { (range) }\end{array}$ & $\begin{array}{l}\text { Initial neck-shaft angle } \\
\text { degrees (range) }\end{array}$ & $\begin{array}{l}\text { Changes in humeral head } \\
\text { height }^{*}, \mathrm{~mm} \text { (range) }\end{array}$ \\
\hline \multirow[t]{3}{*}{ Medial mechanical support of the proximal humerus } & Yes $(n=43)$ & $74.5(40-100)$ & $4.6(1-14)$ & $1.0(0-4.3)$ \\
\hline & No $(n=13)$ & $55.4(46-71)$ & $9.4(2-21)$ & $3.3(0.5-7.2)$ \\
\hline & $p$-value & $<0.001^{\dagger}$ & $<0.001^{\dagger}$ & $<0.001^{\dagger}$ \\
\hline \multirow{3}{*}{$\begin{array}{l}\text { Difference between ipsilateral and contralateral } \\
\text { neck-shaft angles, degrees }\end{array}$} & $\leq 5(\mathrm{n}=35)$ & $78.6(66-100)$ & $3.2(1-7)$ & $0.6(0-1.6)$ \\
\hline & $>5(\mathrm{n}=21)$ & $55.9(40-68)$ & $9.8(2-21)$ & $3.2(0.3-7.2)$ \\
\hline & $p$-value & $<0.001^{\dagger}$ & $<0.001^{\dagger}$ & $<0.001^{\dagger}$ \\
\hline
\end{tabular}

${ }^{*}$ The changes in humeral neck-shaft angles or humeral head heights between immediate postoperative and last follow-up radiographs. ${ }^{\dagger}$ Independent $\mathrm{t}$-test.

outcomes in terms of the Constant score, decrease in humeral neck-shaft angle and the humeral head height at both postoperative and at final follow-up than when the difference was less than $5^{\circ}(p<0.01)$. Similarly, in terms of the restoration of medial mechanical support within the humeral fracture, all three clinical outcomes were better when there was mechanical support than when there was not $(p<0.001)$ (Table 2). When we compared only the 28 patients with fractures containing medial metaphyseal comminution, the 16 patients who had achieved mechanical support of the medial humeral fracture through locking screw fixation and further went on to receive allogenic bone grafting gained a better score in all 3 categories than those who did not achieve mechanical support; Constant score (72.1 vs 54.6, $p<0.001$ ), change in humeral neck-shaft angle (4.9 vs 9.3, $p=0.015$ ), and change in humeral head height (1.2 vs 3.4, $p=0.001)$.

Postoperative complications included one case of intra-articular screw perforation, one case of varus deformity of less than $120^{\circ}\left(111^{\circ}\right)$ with screw loosening, and one case of nonunion. The nonunion of the fracture occurred in an 81-year old patient who was classified with a Neer 2-part fracture. Since the patient was old and the fracture was stabilized, no further surgery was implemented. Other complications such as axillary nerve paralysis, deep infection, and avascular necrosis of the humeral head did not occur.

\section{Discussion}

In our study, the treatment of proximal humeral fractures using locking plates showed encouraging results as in other clinical papers $^{4}$; a high percentage of bone union, a high Paavolainen radiographic score, Constant score of an average 70 points, and a low chance of complications. ${ }^{6-8)}$ However, treatment using locking plates has been shown to be associated with postoperative complications such as avascular necrosis, intra-articular penetration of the locking screws. ${ }^{11,17,18)}$ Our study also described a subset of patients with postoperative complications such as intra- articular perforation of locking screws and non-union. Despite surgical treatment, we also found that 6 patients (11\%) still had a 'poor' Constant score.

Factors related to the patient that may influence the treatment outcomes of proximal humeral fractures include age, sex, fracture type, presence of medial metaphyseal communition in the fracture, and bone density. Likewise, factors related to surgery that may influence the outcome include accuracy of anatomic reduction and restoration of medial mechanical support. ${ }^{11,14,19-22)}$ Krappinger et al. ${ }^{23)}$ analyzed these factors and found that the following factors are important for a successful stabilization of the fracture in the order of anatomic reduction, medial cortical support, proximal humeral bone density, and patient age. The current study also analyzed these factors to determine whether these affect the clinical outcomes. We found that the clinical outcome of treatment was influenced by all factors, excluding bone density.

The restoration of medial mechanical support of the fractures is an important factor that is emphasized for a successful clinical outcome in proximal humeral fractures. ${ }^{14,24,25)}$ Gardner et al. ${ }^{14)}$ showed that when there is an unstable medial mechanical support by comminuted fractures at the proximal humerus, the positioning of the locking screws on the lower side of the humeral head along the humeral calcar is critical. In this study, we also highlighted that patients who showed contact of the medial humerus with the cortical bone or mechanical support of the medial humerus by the locking screws located on the humeral calcar had a greater Constant score, less change in humeral neck-shaft angle, and less change in humeral head height. Especially, for the 28 patients who had medial metaphyseal comminution, an enhanced clinical outcome was seen when treatment was done by fixing locking screws at the humeral calcar for mechanical support. If the locking plates and screws cannot be fixed due to deficit of the posteromedial cortical bone, then a structural bone grafting is required, and for this, the authors used an intramedullary femoral head strut graft augmentation.

The humeral neck-shaft angle is assessed by taking difference 
between the neck-shaft angle measured in the patient after surgery and either the average neck-shaft angle or the neck-shaft angle of the unaffected contralateral arm. In general, the normal neck-shaft angle is considered as $130^{\circ}$ and a neck-shaft angle of below $120^{\circ}$ is thought to be a significant change. Previous studies have found that an accurate anatomical reduction of the humeral fracture is important for a successful clinical outcome using indices such as the position of the greater tubercle, humeral head, humeral shaft, or a post-operative varus angulation of the neck-shaft angle less than $120^{\circ}$ to conclude anatomical reduction. ${ }^{11,23)}$ In this study, since the angle prior to the fracture cannot be known, we concluded anatomic reduction of the neckshaft angle post-surgery using the angle determined from the contralateral side. The standard radiographic assessment using the method by Paavolainen et al. ${ }^{13)}$ was used to measure the absolute angle value. As in other studies we found that an accurate anatomical reduction of the humeral fracture is an important factor for a good clinical outcome.

In this study, the decrease in humeral neck-shaft angle in patients over the age of 65 was significantly greater than in patients under the age of $65(p=0.010)$. Although the Constant score also tended to be lower in the former group than the later, there was no significant difference $(p=0.100)$. However, after further scrutiny of the results, we found that $68 \%$ of patients over the age of 65 (15 out of 22 cases) had medial metaphyseal comminution, but only $38 \%$ of patients over the age of 65 had it ( 13 out of 34 cases). Conversely, the finding was the opposite when only looking at patients with 4-part fractures. Only 5\% (1 case) of patients over the age of 65 with a 4-part fracture had medial metaphyseal comminution, whereas $21 \%$ of patients under the age of 65 with a 4-part fracture had it. This $68 \%$ value is therefore likely to be an underestimate because, all patients over the age of 70 with Neer 4-part fractures underwent artificial joint replacement, and thus were excluded from the study. Therefore, since specific age-groups were excluded from this study, the study is limited in that the results cannot indicate how age influences the surgical outcomes in patients with the same fracture type.

Our data were able to confirm reports of previous studies that found that a low Constant score and a low neck-shaft angle in females, which in turn is related to the incidence of osteoporosis in females, influences the treatment outcome. In conjunction, we found that a significantly lower bone density was detected in females compared to males. However, there was no influence of fracture type and the presence of medial metaphyseal comminution in males and females on the treatment outcome between these two groups.

Osteoporosis is closely related to incidence of proximal humeral fractures and whether the fractures can be stably fixed after treatment. ${ }^{26)}$ However, the current study did not find a significant correlation between osteoporotic bone and the clinical outcomes after a proximal humeral fracture treatment. But limitations to this study such as having a small study population and implementation of osteroporosis test on regions other than the humerus such as the spine and hip bone, which may have not truly reflected the degree of osteoporosis in the humerus. Such limitations may have underestimated the effects of osteoporosis. Further, the difference between the bone density of the spine and/or the hip and the bone density of the proximal humerus may be accounted by the patient's body mass index and muscle strength. Thus, the discrepancy that may be seen between the presence of osteoporosis and the bone densities in the proximal humerus, spine, and hip may be the result of this. ${ }^{27)}$ An alternative method to reveal the influence of osteoporosis on treatment outcomes may have been to use computed tomographic scans to determine cortical thickness as an indicator of osteoporosis in the proximal humerus.

The postoperative complications we observed in patients after treatment of proximal humeral fractures were the perforation of intra-articular locking screws, varus deformity and loosening of the locking screws, and non-union of the bone. All patients were elderly, being of age 86,81 , and 66 years old, respectively. They also had medial metaphyseal comminution and a humeral calcar that was not fixed with locking screws. Further, their fractures were of complex nature, one being a Neer 4-part and two Neer 3-part fractures. Post-surgical outcome was not satisfactory either as these patients had a neck-shaft angle of below $120^{\circ}$ indicating a non-anatomic reduction of the proximal humerus. If in these patients, operative fixation by locking screws at the humeral calcar was performed, anatomical reduction may have been achieved. In general, avascular necrosis is a relatively commonly occurred complication in treating proximal humeral fractures, however in our study none was seen with this complication. ${ }^{17,28)}$ This could be because the elapsed time was short and a preferential treatment of humeral head replacement over locking plates in the patients aged over 70 who had a 4-part fracture.

Overall, the current study has the following limitations. The size of the study population was not sufficient and the period for the follow-up was short. Also, the Constant score that was assigned as a marker for the functional outcome does not reflect the influence of age on the change in the range of motion or in muscle strength. Further, even though a difference may exist between the two methods, mechanical support by locking screws and allogenic bone grafting were considered as the same, and so, the difference in the clinical outcome resulting from these two types of methods was not investigated. In addition, the bone density analysis we performed reflects osteoporosis in general and not specifically on bone densities of proximal humeral fractures. As the tests for osteoporosis was taken at the spine, hip, and wrists, not at the proximal humerus, thus approach to assess osteoporosis may have reliably reveal the effect of osteoporosis as one factor influencing the clinical outcomes of proximal hu- 
meral fracture treatment. Lastly, a multivariate analysis of factors was not carried out.

The clinical outcomes of operative fixation of proximal humeral fractures using locking plates were generally successful. However, in our study, this was not the case for patients with 4-part fractures (Neer classification), C-type fractures (AO classification), medial comminuted fractures, or for female patients. In these patients a less favorable clinical outcome was seen. To achieve better clinical outcomes in such patients, an accurate reduction of the fracture and the medial mechanical support is needed. Further, if mechanical support can be achieved at the region of fracture by fixing the locking screws at the humeral metaphyseal calcar may give good clinical results even in the presence of medial metaphyseal comminution.

\section{References}

1. Court-Brown CM, Caesar B. Epidemiology of adult fractures: $A$ review. Injury. 2006;37(8):691-7.

2. Oh JH, Kim YH. The current concepts in the treatment of proximal humerus fracture. J Korean Fract Soc. 2012;25(1): 94-104.

3. Baron JA, Karagas M, Barrett J, et al. Basic epidemiology of fractures of the upper and lower limb among Americans over 65 years of age. Epidemiology. 1996;7(6):612-8.

4. Björkenheim JM, Pajarinen J, Savolainen V. Internal fixation of proximal humeral fractures with a locking compression plate: a retrospective evaluation of 72 patients followed for a minimum of 1 year. Acta Orthop Scand. 2004;75(6):741-5.

5. Egol KA, Kubiak EN, Fulkerson E, Kummer FJ, Koval KJ. Biomechanics of locked plates and screws. J Orthop Trauma. 2004;18(8):488-93.

6. Koukakis A, Apostolou CD, Taneja T, Korres DS, Amini A. Fixation of proximal humerus fractures using the PHILOS plate: early experience. Clin Orthop Relat Res. 2006;442:115-20.

7. Park $\mathrm{CH}$, Park $\mathrm{SH}$, Seo JS. Internal fixation of proximal humerus fracture with locking compression plate. J Korean Shoulder Elbow Soc. 2009;12(1):44-52.

8. Ha SS, Kim JY, Hong KD, Sim JC, Kang JH, Park KH. Operative treatment with locking compression plate (LCP) in proximal humerus fracture. J Korean Shoulder Elbow Soc. 2008;11(2):137-42.

9. Siwach R, Singh R, Rohilla RK, Kadian VS, Sangwan SS, Dhanda $M$. Internal fixation of proximal humeral fractures with locking proximal humeral plate (LPHP) in elderly patients with osteoporosis. J Orthop Traumatol. 2008;9(3):149-53.

10. Plecko M, Kraus A. Internal fixation of proximal humerus fractures using the locking proximal humerus plate. Oper Orthop Traumatol. 2005;17(1):25-50.

11. Agudelo J, Schürmann M, Stahel $P$, et al. Analysis of efficacy and failure in proximal humerus fractures treated with locking plates. J Orthop Trauma. 2007;21(10):676-81.

12. Constant CR, Murley AH. A clinical method of functional assessment of the shoulder. Clin Orthop Relat Res. 1987;(214):160-4.

13. Paavolainen P, Björkenheim JM, Slätis P, Paukku P. Operative treatment of severe proximal humeral fractures. Acta Orthop Scand. 1983;54(3):374-9.

14. Gardner MJ, Weil Y, Barker JU, Kelly BT, Helfet DL, Lorich DG. The importance of medial support in locked plating of proximal humerus fractures. J Orthop Trauma. 2007;21(3):185-91.

15. Shrout PE, Fleiss JL. Intraclass correlations: uses in assessing rater reliability. Psychol Bull. 1979;86(2):420-8.

16. Walsh S, Reindl R, Harvey E, Berry G, Beckman L, Steffen T. Biomechanical comparison of a unique locking plate versus a standard plate for internal fixation of proximal humerus fractures in a cadaveric model. Clin Biomech. 2006;21(10):102731.

17. Spross C, Platz A, Rufibach K, Lattmann T, Forberger J, Dietrich $M$. The PHILOS plate for proximal humeral fractures--risk factors for complications at one year. J Trauma Acute Care Surg. 2012;72(3):783-92.

18. Fankhauser F, Boldin C, Schippinger G, Haunschmid C, Szyszkowitz R. A new locking plate for unstable fractures of the proximal humerus. Clin Orthop Relat Res. 2005;(430):17681.

19. Hertel R. Fractures of the proximal humerus in osteoporotic bone. Osteoporos Int. 2005;16 Suppl 2:S65-72.

20. Micic ID, Kim KC, Shin DJ, et al. Analysis of early failure of the locking compression plate in osteoporotic proximal humerus fractures. J Orthop Sci. 2009;14(5):596-601.

21. Röderer G, Erhardt J, Kuster M, et al. Second generation locked plating of proximal humerus fractures--a prospective multicentre observational study. Int Orthop. 2011;35(3):42532.

22. Solberg BD, Moon CN, Franco DP, Paiement GD. Locked plating of 3- and 4-part proximal humerus fractures in older patients: the effect of initial fracture pattern on outcome. J Orthop Trauma. 2009;23(2):113-9.

23. Krappinger D, Bizzotto N, Riedmann S, Kammerlander C, Hengg C, Kralinger FS. Predicting failure after surgical fixation of proximal humerus fractures. Injury. 2011;42(11):1283-8.

24. Osterhoff G, Hoch A, Wanner GA, Simmen HP, Werner CM. Calcar comminution as prognostic factor of clinical outcome after locking plate fixation of proximal humeral fractures. Injury. 2012;43(10):1651-6.

25. Robinson CM, Wylie JR, Ray AG, et al. Proximal humeral fractures with a severe varus deformity treated by fixation with a locking plate. J Bone Joint Surg Br. 2010;92(5):672-8.

26. Lee SH, Dargent-Molina P, Bréart G; EPIDOS Group. Epidemiologie de I'Osteoporose Study. Risk factors for fractures of the proximal humerus: results from the EPIDOS prospective study. 
J Bone Miner Res. 2002;17(5):817-25.

27. Doetsch AM, Faber J, Lynnerup N, Wätjen I, Bliddal H, Danneskiold-Samsøe B. Bone mineral density measurement over the shoulder region. Calcif Tissue Int. 2002;71(4):308-14.
28. Moonot P, Ashwood N, Hamlet M. Early results for treatment of three- and four-part fractures of the proximal humerus using the PHILOS plate system. J Bone Joint Surg Br. 2007;89(9):1206-9. 